\section{A Quantitative Approach to Human Issues in Horticulture: Conjoint Analysis}

\author{
Wilmien Brascamp ${ }^{1}$
}

\begin{abstract}
ADDITIONAL INDEX wORDs. consumer research, landscape preference, people-plant interactions

SumMary. Research on human issues in horticulture focuses on the human dimension of horticulture in an effort to maximize the benefits of plants and nature in general, for human well-being. A key issue is the need for scientific evidence of such benefits and for rigorous research methods to reveal the mechanics of the interaction between people and plants. Conjoint analysis, a methodology with obvious potential for successful application in the area of human issues in horticulture, is widely used in consumer research to estimate the structure of people's reactions to multi-attribute objects or services. This paper discusses the steps involved in implementing conjoint analysis and describes how it can be applied to people-plant research.
\end{abstract}

$\mathrm{D}$ ata supported by sound science must be provided for people-plant research to reach its full potential, and for people-plant research findings to be applied in practice (Frumpkin, 2004; Owen, 1994). Whilst horticulturists, with their indepth knowledge and understanding of plant-related aspects of interactions between people and plants could make important contributions here, their involvement to date in people-plant research has been limited (Shoemaker et al., 2000). Lack of familiarity among horticulturists with research methodologies for conducting people-plant research has been suggested as a major limiting factor.

Shoemaker et al. (2000) urged horticulturists with an interest in people-plant interactions to explore possibilities offered by social science methodologies, and thus create the opportunity to position themselves at the forefront of research on human issues in horticulture. Conjoint analysis is one such methodology with obvious potential for successful applications across the entire field of human issues in horticulture. This powerful analytic technique is currently the most commonly used research method for unraveling people's perceptions of multi-attribute products and services (Green et al., 2001). Since developments in mathematical psychology led to its conceptual framework (Luce and Tukey, 1964), the conjoint methodol-

\footnotetext{
${ }^{1}$ Landscape Group, Institute of Natural Resources -PN433, Massey University, Private Bag 11222 , Palmerston North 5301, New Zealand.
}

ogy has received widespread attention from academia (Green and Srinivasan, 1978,1990 ) and practitioners (Cattin and Wittink, 1982; Wittink and Cattin, 1989; Wittink et al., 1994).

In response to the need for rigorous scientific investigations of people-plant interactions, and supporting the view that horticulturists have an important role to play in such research (Shoemaker et al., 2000), this paper explains the principles and underlying concepts of conjoint analysis for application in the area of human issues in horticulture. The objective is to provide a framework for conducting conjoint analysis projects in the area of people-plant interactions, drawing on a conjoint study of people's evaluations of outdoor ornamental plants (Brascamp, 1996) to illustrate the process. Further application opportunities for the methodology in people-plant research are suggested to indicate its wide-ranging potential.

While the focus is on one specific technique, the material presented here also serves a more general aim; that of encouraging people-plant researchers to explore techniques that are successfully applied in other research areas.

\section{Basic ideas of conjoint analysis}

Conjoint analysis is based on people's evaluations of factorially designed product or service alternatives (Carroll and Green, 1995). A typical conjoint analysis study begins with determining the product or service attributes to be included, followed by specifying attribute levels. Attributes are product or service characteristics, such as price, color, styling, and speed of service delivery. The selected attributes are then treated as factors in a factorial design, each occurring at a range of levels. Each treatment combination constitutes a product or service profile, defined by a particular set of attribute levels. Profiles are represented as illustrations, descriptions, actual objects, or product concepts. Data are collected from respondents who are asked to evaluate the profiles, for example, by rating them on a predetermined preference scale. In most marketing applications of conjoint analysis, evaluations are related to perceived product quality or intention to purchase. In general however, evaluations may be of various kinds, ranging from attractiveness ratings to physiological or psychological responses. People's evaluations of, or responses to, any multi-attribute object, scene, service or task can be investigated with conjoint analysis. Respondent evaluations serve as input for an analysis of variance type of approach. Using respondent evaluations of profiles as the dependent variable, conjoint measurement studies reveal to what extent individual attributes influence respondents' overall evaluations. Thus, conjoint analysis of people's evaluations of alternative profiles provides insight into the make-up of their overall evaluations, dealing with the question why certain profiles are preferred over others. Detailed overviews of conjoint analysis are presented by Curry (1997), Green et al. (2001), Gustafsson et al. (2001), and McCullough (2002).

\section{Selection of attributes and specification of attribute levels}

Since conjoint studies are based on a factorial design and the number of treatment combinations increases exponentially as more attributes are included, the number of attributes and their levels that can be taken into account may have to be limited to avoid information overload for respondents (Green and Srinivasan, 1978). When making choices between different brands or products in real life situations, people often consider a limited set of alternatives, and evaluate each alternative on a subset of attributes (Lussier and Olshavsky, 1979).

Preliminary research is necessary to gain some insight into such subsets of attributes and for the conjoint data 
collection phase to result in meaningful and reliable information. In her study on garden center customer evaluations of outdoor ornamental plants, Brascamp (1996) conducted preliminary interviews, questioning garden center customers regarding attributes of outdoor plants that are important to them when making a purchase selection. Eight plant attributes were thus suggested for inclusion in the conjoint study: price, final height, health, suitability for the respondent's garden, shape, flower color, bushiness, and leaf color. Three levels were selected for the first four attributes, and two levels were assigned to each remaining plant attribute. For example, three final height levels were specified: $<1 \mathrm{~m}, \mathrm{l}-2$ $\mathrm{m}$, and $>2 \mathrm{~m}(1 \mathrm{~m}=3.2808 \mathrm{ft})$. Dark green and light green represented the two levels assigned to the attribute "leaf color". Elicitation interviews avoid researcher bias in the attribute selection process, minimize the risk of including irrelevant attributes, and elicit attributes and attribute levels in terms commonly used, and therefore easily understood, by respondents (Louviere, 1988).

\section{Experimental design}

Various data collection procedures can be used for conjoint analysis (Green et al., 2001; McCullough, 2002), using full profiles that each represent the full set of attributes accounted for in the study, or partial profiles where each represents a different subset of attributes included in the study. Thus, in the full-profile procedure, profile alternatives are presented in a more realistic way, utilizing all attributes under study, with the attribute level combinations in each profile being determined by a factorial design (Green and Srinivasan, 1978). Maximum information in a full-profile study is theoretically obtained with a complete factorial design. However, as the number of profiles an individual respondent is required to evaluate, increases, the reliability of the information obtained decreases due to information overload on the part of the respondent (Green and Srinivasan, 1978). Since people's preferences are commonly influenced by the joint effect of "more" rather than "few" product attributes, conjoint research design usually necessitates a trade-off between information and reliability. For example, a complete factorial design for a full-profile study, such as the one conducted by Brascamp (1996), with eight attributes, four at three levels and four at two levels, would require each respondent to evaluate $3^{4} \times 2^{4}(=1296)$ profiles, clearly an unrealistic task. The essential feature of conjoint analysis is estimation of the quantitative effects of product attributes and attribute levels on respondents' evaluations using fractional factorial designs, whereby fewer than all possible profiles are presented to respondents. Thus, each respondent is asked to undertake a realistic task (evaluate a relatively small number of profiles), whilst the researcher recognizes potential information loss for the sake of increased reliability about those effects estimated. Comprehensive discussions on the theory and practice of developing fractional factorial designs, either manually, or to select and adapt, where necessary, computer-generated fractions, are available (Gunst and Mason, 1991; Hinkelmann and Kempthorne, 1994).

In fractional factorial designs, some high-order interaction effects, such as effects of interactions between three or more attributes, are confounded with some low-order effects, such as main effects or effects of interactions between two attributes, on the assumption that those confounded high-order effects are negligible relative to explaining response variable variance. The result is a reduction in the number of profiles required to estimate effects of attributes and attribute interactions that are considered to be of likely significance. Confounding high-order interaction effects implies a loss of information, since their effects can no longer be estimated. However, since the proportion of total variance in responses accounted for by interactions between three attributes seldom exceeds $2 \%$ to $3 \%$, and higher-order interaction terms explain even less of the variation (Louviere, 1988), the extensive use by conjoint analysts of fractions of factorial designs that confound some such interactions with lower-order interactions is justified.

Preliminary research may provide justification for trade-offs between low-order interactions, if there are strong indications that some low-order interactions are more important than others in explaining response variable variance. In the study of Brascamp (1996) with four three-level attributes and four two-level attributes, and thus
$3^{4} \times 2^{4}(=1296)$ treatment combinations (profiles), results of preliminary interviews indicated that only a limited number of interactions would likely be of importance in affecting consumers' overall evaluations of outdoor plants. The attribute "price" was expected to interact with each remaining attribute. It also seemed plausible to assume that consumer preference for a particular leaf color depends on the brightness of the flowers. Likewise, the attributes "shape" and "bushiness" were expected to interact. Assuming that remaining interaction effects were negligible, their terms were used for the development of a fraction of the complete factorial design and subsequently for the specification of blocks within this fraction.

Since, in a fractional factorial design, each respondent is to evaluate only a relatively small number of profiles, this set can be considered as a "block" in classical experimental design terminology. These block, or respondent, effects can in turn be confounded with interaction effects that are considered to be non-significant, thus further reducing the number of profiles in the set to be evaluated by a respondent.

A replicate of the fractional factorial design will consist of the responses of $x$ respondents, each evaluating $y$ different profiles. The efficiency with which the selected effects are estimated, can be increased by using a number of replicates of the basic fractional factorial design. As the number of replicates is increased, the variance of the estimated effects decreases. The possibility now arises that different confounding arrangements can be used in different replicates, thus increasing overall the number of effects that can be estimated, but with a consequent loss of efficiency with which at least some effects are estimated. Whilst this possibility is not explored further here, Brascamp (1996) took advantage of this opportunity in her conjoint research. The resulting fractional factorial design consisted of 288 sets of six different treatment combinations (plant profiles), arranged in eight replicates, each using a different confounding arrangement, and each replicate consisting of 36 blocks (respondents) of six treatments per block; hence 1728 responses from 288 respondents. The basic design was then repeated six times to give a total of 10368 response observations. 


\section{Profile presentation}

Once a design is constructed, the profiles can be developed. Treatment combinations can be presented in verbal, pictorial, or combined formats. In some cases, for example, in the study on recreational value of rural areas by Goossen and Langers (2000), with attributes such as noise levels and accessibility, the verbal format may be the only practical option. The textual approach, where respondents are presented with stimulus cards on which the levels of each attribute are defined in words, is simple and often efficient in data collection. Visual stimuli on the other hand, more closely resemble reality, positively affecting the validity of responses, in particular where product styling or design is an important aspect of the research (Loosschilder et al., 1995). Responses to landscapes and environmental evaluation (Earnhart, $2001)$ generally fall into this category. Moreover, pictorial presentations of treatment combinations are likely to reduce the complexity of the task facing individual respondents. Such presentations require a different, simpler information processing system than do verbal profiles that need to be read and subsequently visualized (Green and Srinivasan, 1978). Comparing different visual formats, Jaeger et al. (2001) suggested that photographic images, instead of physical prototype stimuli, are appropriate to represent alternatives of well-known products or objects.

Brascamp (1996)designed profiles using a combination of visual and verbal information. Profile cards consisted of a hypothetical plant, illustrated in terms of five of the eight attributes (health, shape, bushiness, flower color, and leaf color), and included descriptions of the remaining attributes that did not lend themselves for visual presentation (price, final height, and suitability for the respondent's garden).

\section{The estimated model}

The basis for models proposed for application in conjoint measurements, is referred to by Green and Srinivasan (1978) as the part-worth model, and can be expressed as follows:

$$
\Upsilon_{j}=\sum_{p=1}^{t} f_{p}\left(x_{j p}\right)
$$

where $\Upsilon_{j}$ is an individual's evaluation of the $j$ th profile; $x_{j p}$ is the level of the $p$ th attribute for the $j$ th profile; $f_{p}$ is a function denoting the "part worth" of level $x_{j p}$ for the $p$ th attribute of $t$ attributes. The outcome of this model is a set of part-worths (numerical expressions) of the contribution of each attribute level to the overall evaluation. Part-worths so obtained can be used to estimate the overall evaluation of a profile representing any combination of attribute levels.

The model can readily be extended to account for effects of attribute interactions. If, for example, variations in profile evaluations were completely accounted for by effects and interactions of two attributes sand $t$, each with two levels, then, assuming an additive function of judgments, an individual's evaluation of a profile is described by the following model:

$$
\Upsilon_{i j}=\mu+S_{i}+T_{j}+(S T)_{i j}
$$

where $\Upsilon_{i j}$ is the evaluation of profile $i j ; \mu$ is the mean of profile evaluations; $S_{i}$ and $T_{j}$ estimate main effects of attributes $s$ and $t ;(S T)_{i j}$ estimates the interaction effects between attributes $s$ and $t$.

\section{Data collection and analysis}

In full-profile conjoint studies data can be collected using either a ranking or a rating scale. In ratings-based conjoint approaches, respondents evaluate profiles on a predetermined scale, such as a likelihood-of-purchase scale, or an attractiveness scale. When using a ranking scale, respondents are required to rank profiles in order of preference. Rating scales have become more popular than rank orders in commercial applications of conjoint analysis. This is possibly due to the confidence of conjoint analyzers in the metric qualities of data generated by ratings-based conjoint studies, and therefore, in the appropriateness of analysis of variance (ANOVA) approaches (Wittink et al., 1994). Whereas rated profile evaluations express the intensity of preferences, ranked data just represent an order of preferences (Gustafsson et al., 2001). Whilst there are software packages available that have been designed specifically for ratings- and rankings-based conjoint analysis, such as from Sawtooth Software (2002) and SPSS Inc. (2004), metric full-profile conjoint data may serve as input for any statistical package that can perform an ANOVA.

Evidence suggests that the perfor- mance of full-profile conjoint analysis in terms of its predictive ability is improved by "warm-up" tasks prior to actual conjoint data collection (Huber et al., 1993). Warm-up exercises or training procedures allow respondents to familiarize themselves with attributes and their levels included in the conjoint task, and with the presentation format. This may be particularly important when studying topics other than non-durable consumer goods, that are unfamiliar to respondents, and for which purchase decisions or other evaluative judgments are made infrequently (Jaeger et al., 2001), such as environmental evaluation. In her warm-up exercise, Brascamp (1996) presented respondents with cards on which individual plant attributes and levels were described and illustrated where appropriate. Respondents were asked to rank the levels of each attribute in order of preference, and to indicate the importance of each attribute in determining the choice for a particular plant. For the conjoint data collection phase, respondents were asked to rate each of six plant profiles on a scale from 1 (very attractive) to 7 (very unattractive).

A variety of parameter estimation procedures exists for conjoint analysis (Carroll and Green, 1995; Green et al., 1993). In full-profile conjoint studies, evaluations typically serve as input for an ANOVA or a variant thereof. The model specifying the relationship between dependent and independent variables when assuming an additive function of effects can be written in matrix and vector notation as follows:

$$
\Upsilon=X \beta+\varepsilon
$$

where $\Upsilon$ is the vector of observations (evaluations); $X$ is the design matrix; $\beta$ is the vector of unknown parameters; and $\varepsilon$ is the vector of error terms.

Brascamp (1996) used the General Linear Model procedure of SAS that routinely solves the normal equation $X^{\prime} X b=X^{\prime} \Upsilon$, producing a generalized inverse $\left(X^{\prime} X\right)^{-1}$ and a solution $b$ for $\beta$, which equals $\left(X^{\prime} X\right)^{-1} X^{\prime} \Upsilon$ (SAS Institute, 1989). ANOVA results provided statistical evidence that effects of all plant attributes included in the study were significant $(P<0.05)$. Preference for a particular price level depended on the health status, the final height, the suitability for the respondent's garden, 
and on the shape of the plant. A significant interaction also existed between the shape and the degree of bushiness of a plant $(P<0.05)$. In an ANOVA, numerical values are assigned to each level of each attribute and interaction. For example, the effect of a poor health status, expressed as a deviation from the overall mean rating of $\mu=3.3$, was -1.1 . The effect of a good health status on people's profile evaluations was +0.9 . Results can be used to project evaluations of any combination of attributes, so that one could, for example, calculate which profile would elicit the most positive response.

Hays' omega squared values (Green et al., 1988; Hays, 1963) were calculated for significant effects and interactions to measure the strength of association between these and the response variable. Calculating omega squared values as proportions of the total variation accounted for by significant effects and interactions, the attribute "health" accounted for $38.7 \%$ of all variation. The attribute "health" was followed in importance by the attributes "suitability for the respondent's garden" (29.4\%), "shape of the plant" (14.6\%), "bushiness" (6.8\%), "price" $(3.6 \%)$, by the interaction between "price" and "final height" (3.4\%), and finally by the attribute "final height" (1.7\%).

In summary, the analysis reveals to what extent attributes and their interactions influence people's evaluations, and estimates the contribution of each attribute level to people's overall evaluation of a product or service.

\section{Applications of conjoint analysis in research on human issues in horticulture}

Conjoint analysis lends itself for application in each of the five key research areas identified by researchers and practitioners in the field of human issues in horticulture. These areas include human well-being, people-plant interactions in urban settings, children-plant interactions, physical and perceived quality of the environment, and economic aspects of relationships between plants and people (Lohr and Relf, 1993). In each priority area, the core question pertains to people's responses to plants. Conjoint analysis provides a methodological framework for quantifying such responses, thus leading to a greater understanding of the way in which plants can be used and landscapes can be designed, for the benefit of humanity. The decompositional nature of conjoint models overcomes an important dilemma facing researchers in the field of human issues in horticulture: that, while people generally have no difficulty expressing their preference level for a scene or a product, they are often unable to identify reasons for their preferences.

Conjoint analysis input is not confined to people's evaluations of certain plant products, landscapes or horticultural services, but can be extended to include physiological responses, for example by using blood pressure or heart rate measurements as the dependent variable in the conjoint analysis model. Conjoint measurement can also be applied to study people's emotions evoked by certain stimuli (Kellaris and Kent, 1994). Effects of plants on people are well documented (Lohr and Relf, 2000) and several theories have been developed to explain such influences (Relf, 1999). Conjoint analysis offers an additional tool to take human issues in horticulture beyond the stage of identifying the benefits of nature, to the level of quantifying the relative contributions to these overall benefits of specific aspects of people-plant interactions. As such, application of conjoint analysis in people-plant studies will contribute to the development and establishment of this relatively young research field as an area of rigorous scientific investigation with far-reaching benefits for humanity.

\section{Literature cited}

Brascamp, W. 1996. Evaluation and measurement of consumer preferences for outdoor ornamental plants. PhD Diss., Massey Univ., Palmerston North, New Zealand.

Carroll, J.D. and P.E. Green. 1995. Psychometric methods in marketing research: Part 1, Conjoint analysis. J. Mktg. Res. 32(4):385-391.

Cattin, P. and D.R. Wittink. 1982. Commercial use of conjoint analysis: A survey. J. Mktg. 46(3):44-53.

Curry, J. 1997. After the basics. Mktg. Res. 9(1):6-11.

Earnhart, D. 2001. Combining revealed and stated preference methods to value environmental amenities at residential locations. Land Econ. 77(1):12-29.
Frumpkin, H. 2004. White coats, green plants: Clinical epidemiology meets horticulture. Acta Hort. 639:15-26.

Goossen, M. and F. Langers. 2000. Assessing quality of rural areas in the Netherlands: Finding the most important indicators for recreation. Landscape Urban Planning 46:24l-25l.

Green, P.E. and V. Srinivasan. 1978. Conjoint analysis in consumer research: Issues and outlook. J. Consumer Res. 5:103-123.

Green, P. E. and V. Srinivasan. 1990. Conjoint analysis in marketing: New developments with implications for research and practice. J. Mktg. 54(4):3-19.

Green, P.E., D. Tull, and G. Albaum. 1988. Research for marketing decisions. 5th ed. Prentice Hall, Englewood Cliffs, N.J.

Green, P.E., A.M. Krieger, and Y. Wind. 2001. Thirty years of conjoint analysis: Reflections and prospects. Interfaces 31 [ 3 (part 2)]:56-73.

Green, P.E., A.M. Krieger, and C.M. Schaffer. 1993. A hybrid conjoint model with individual-level interaction estimation. Adv. Consumer Res. 20:149-154.

Gunst, R.F. and R.L. Mason. 1991. How to construct fractional factorial experiments. Amer. Soc. Quality Control, ASQC Quality Press, Milwaukee, Wis.

Gustafsson, A., A. Herrmann, and F. Huber. 2001. Conjoint analysis as an instrument of market research practice, p. 5-46. In: A. Gustafsson, A. Herrmann, and F. Huber (eds.). Conjoint measurement. Methods and applications. 2nd ed. Springer-Verlag, New York.

Hays, W.L. 1963. Statistics for psychologists. Hold, Rinehart and Winston, New York.

Hinkelmann, K. and O. Kempthorne. 1994. Design and analysis of experiments. Wiley, New York.

Huber, J., D.R. Wittink, J.A. Fiedler, and R. Miller. 1993. The effectiveness of alternative preference elicitation procedures in predicting choice. J. Mktg. Res. 30:105-114.

Jaeger, S.R., D. Hedderley, and H.J.M. MacFie. 2001. Methodological issues in conjoint analysis: A case study. European J. Mktg. 35(11/12):1217-1239.

Kellaris, J.J. and R.J. Kent. 1994. An exploratory investigation of responses elicited by music varying in tempo, tonality, and texture. J. Consumer Psychology 2(4):381-401.

Lohr, V.I. and D. Relf. 1993. Human issues in horticulture: Research priorities. 
HortTechnology 3(1):106-107.

Lohr, V.I. and P.D. Relf. 2000. An overview of the current state of human issues in horticulture in the United States. HortTechnology 10(1):27-33.

Loosschilder, G.H., E. Rosbergen, M. Vriens, and D.R. Wittink. 1995. Pictorial stimuli in conjoint analysis-To support product styling decisions. J. Mkt. Res. Soc. 37(1):17-34.

Louviere, J.J. 1988. Analyzing decision making: Metric conjoint analysis. Sage, Beverly Hills, Calif.

Luce, R.D. and J.W. Tukey. 1964. Simultaneous conjoint measurement: A new type of fundamental measurement. J. Mathematical Psychology 1:1-27.

Lussier, D.A. and R.W. Olshavsky. 1979. Task complexity and contingent processing in brand choice. J. Consumer Res. 6:154-165.

McCullough, D. 2002. A user's guide to conjoint analysis. Mktg. Res. 14(2):1923.

Owen, P.J. 1994. Translating the healing dimensions of plants into scientific terminology, p. 337. (Abstr.) In: M. Francis, P. Lindsey, and J. Stone Rice (eds.). The healing dimensions of people-plant interactions: Proc. Res. Symp., Univ. of Calif., Davis, 24-27 Mar.1994.
Relf, P.D. 1999. The role of horticulture in human well-being and quality of life. J. Therapeutic Hort. 10:10-14.

SAS Institute Inc. 1989. The GLM procedure, p. 891-996. In: SAS/STAT user's guide 2. 4th ed. SAS Inst., Cary, N.C.

Sawtooth Software. 2002. Conjoint Value Analysis 3.0. Sawtooth Software, Sequim, Wash.

Shoemaker. C.A., P.D. Relf, and V.I. Lohr. 2000. Social science methodologies for studying individuals' responses in human issues in horticulture research. HortTechnology 10(1):87-93.

SPSS. 2004. SPSS Conjoint 13.0: add-on module for SPSS 13.0 for Windows. SPSS Inc., Chicago.

Wittink, D.R. and P. Cattin. 1989. Commercial use of conjoint analysis: An update. J. Mktg. 53(3):91-96.

Wittink, D.R., M. Vriens, and W. Burhenne. 1994. Commercial use of conjoint analysis in Europe: Results and critical reflections. Intl. J. Res. Mktg. 11:41-52. 